Journal of Patient-Centered

\title{
Can Magnetic Resonance Imaging Predict Pathologic Findings for Endometrioid Endometrial Cancer?
}

\author{
Elizabeth L. Dickson Michelson \\ Jessica J. F. Kram \\ Kayla Heslin \\ David Baugh \\ Vikram Bamra \\ Jiahao Hu \\ Abhishek Shukla \\ Scott A. Kamelle
}

Follow this and additional works at: https://aah.org/jpcrr

Part of the Analytical, Diagnostic and Therapeutic Techniques and Equipment Commons, Female Urogenital Diseases and Pregnancy Complications Commons, Neoplasms Commons, Obstetrics and Gynecology Commons, Oncology Commons, and the Women's Health Commons

\section{Recommended Citation}

Dickson Michelson EL, Kram JJ, Heslin K, Baugh D, Bamra V, Hu J, Shukla A, Kamelle SA. Can magnetic resonance imaging predict pathologic findings for endometrioid endometrial cancer? J Patient Cent Res Rev. 2020;7:206-12. doi: 10.17294/2330-0698.1720

Published quarterly by Midwest-based health system Advocate Aurora Health and indexed in PubMed Central, the Journal of Patient-Centered Research and Reviews (JPCRR) is an open access, peer-reviewed medical journal focused on disseminating scholarly works devoted to improving patient-centered care practices, health outcomes, and the patient experience. 


\title{
Can Magnetic Resonance Imaging Predict Pathologic Findings for Endometrioid Endometrial Cancer?
}

\author{
Elizabeth L. Dickson Michelson, MD, ${ }^{1}$ Jessica J. F. Kram, MPH, ${ }^{2,3}$ Kayla Heslin, MPH, ${ }^{2,3,4}$ David \\ Baugh, MD, ${ }^{5}$ Vikram Bamra, MD, ${ }^{5}$ Jiahao Hu, MD, ${ }^{5}$ Abhishek Shukla, MD, ${ }^{6}$ Scott A. Kamelle, MD ${ }^{7}$ \\ ${ }^{1}$ Oncology, Aurora West Allis Medical Center, West Allis, WI; ${ }^{2}$ Aurora UW Medical Group, Aurora Health Care, \\ Milwaukee, WI; ${ }^{3}$ Center for Urban Population Health, Milwaukee, WI; ${ }^{4}$ Aurora Research Institute, Aurora Health Care, \\ Milwaukee, WI; ${ }^{5}$ Radiology, Aurora St. Luke's Medical Center, Milwaukee, WI; ${ }^{6}$ Pathology, Aurora St. Luke's Medical \\ Center, Aurora Health Care, Milwaukee, WI; ${ }^{7}$ Oncology, Aurora St. Luke's Medical Center, Milwaukee, WI
}

\begin{abstract}
This pilot study aimed to assess the feasibility of precisely measuring tumor diameter and myometrial invasion in patients with endometrioid endometrial cancer (EEC) using preoperative contrastenhanced magnetic resonance imaging (MRI). Adult patients with confirmed diagnosis of complex hyperplasia with atypia or EEC were included. Three radiologists separately measured tumor diameter and myometrial invasion. Basic descriptive statistics were used to describe patient characteristics and to compare radiology- and pathology-measured tumor diameter and myometrial invasion. Using the pathology results for tumor diameter as the gold standard for comparison, at least 1 radiologist was able to predict largest tumor diameter within $5 \mathrm{~mm}$ for $41.7 \%$ of patients. Similarly, based on pathology results for myometrial invasion, at least 1 radiologist was able to predict myometrial invasion within $5 \%$ for $50 \%$ of patients. All radiologists were able to predict superficial $(<50 \%)$ or deep $(\geq 50 \%)$ myometrial invasion for $75 \%$ of patients, with greater sensitivity, specificity, and accuracy for deep myometrial invasion. Given variation among radiologic measurements, it is difficult to recommend preoperative MRI as a basis for measuring tumor diameter and myometrial invasion. Even so, the ability to predict superficial versus deep myometrial invasion may benefit patients with EEC for whom surgery is not a viable option or for those seeking fertility-sparing treatment options. (J Patient Cent Res Rev. 2020;7:206-212.)
\end{abstract}

Keywords magnetic resonance imaging; endometrioid endometrial cancer; tumor diameter; myometrial invasion

$\mathrm{I}$ $\mathrm{n}$ the United States, endometrial cancer is the most common gynecologic cancer and is one of the few cancers in which incidence is increasing. ${ }^{1-3}$ According to the American Cancer Society, more than 65,000 new cases of endometrial cancer and uterine sarcomas will be diagnosed in 2020, and more than 12,000 women will die from the disease. ${ }^{1}$ While there are other risk factors for developing endometrial cancer, unopposed estrogen is the main factor associated with disease. ${ }^{4,5}$ This can be secondary to the increasing incidence of obesity, in which obese women have

Correspondence: Elizabeth L. Dickson Michelson, MD, Aurora West Allis Medical Center, 8901 W. Lincoln Ave., West Allis, WI 53227 (elizabeth.dicksonmichelson@aah.org) higher levels of endogenous estrogen. ${ }^{4,5}$ Approximately $5 \%$ of patients diagnosed with endometrial carcinoma or atypical hyperplasia are under the age of $40,{ }^{6}$ with findings from Gonthier et al concluding that younger patients are significantly more likely to be obese. ${ }^{5}$ As obesity increases among younger patient populations, more focus is moving toward fertilitysparing treatments for both atypical hyperplasia of the endometrium (a precursor of endometrial cancer) and endometrial cancer itself. ${ }^{5}$

Currently, hysterectomy and lymphadenectomy are recommended for patients with endometrioid endometrial cancer (EEC) who are at greater than low risk of lymphatic dissemination, with hysterectomy only recommended for patients at low risk. ${ }^{2}$ In recent years, traditional pelvic with or without paraaortic 
lymph node dissection has become controversial given increased morbidity. ${ }^{7}$ The Society of Gynecologic Oncology has identified sentinel lymph node mapping as a reasonable technique to map the lymphatic system and identify the first lymphatic drainage for the uterus. ${ }^{7}$ The theory, supported by multiple studies, suggests that these first lymph nodes have a high sensitivity and negative predictive value. ${ }^{89}$ Even so, lymphocysts, lymphedema, and neuralgia are still risk factors associated with lymph node dissection, especially full lymph node dissection. ${ }^{7}$ Given these risks, and the need for fertility-sparing treatment options, developing preoperative screening tools may help determine who should or shouldn't undergo lymphadenectomy or hysterectomy.

Our previously published risk schema, as well as risk schemas from other institutions, used tumor diameter (TD) and myometrial invasion (MI) to predict lowrisk patients who most likely will not benefit from lymphadenectomy. ${ }^{2,10-12}$ Several studies have evaluated magnetic resonance imaging (MRI) for measuring MI, describing variable sensitivity (69\%-94\%), specificity (57\%-86\%), and accuracy (66\%-90\%) when measuring superficial $(<50 \%)$ versus deep $(\geq 50 \%)$ invasion. ${ }^{13-16}$ Some studies have evaluated contrast-enhanced MRI for measuring TD but not as it relates to sensitivity, specificity, and accuracy. ${ }^{17-19} \mathrm{We}$ hypothesized that use of MRI may accurately measure TD and MI preoperatively and may help with the identification of low-risk patients.

This pilot study aimed to assess the feasibility of precisely measuring TD and MI in patients with EEC using preoperative contrast-enhanced MRI. Ultimately, we hoped to determine whether preoperative MRI could be used to save patients from lymphadenectomy or, among patients for whom surgery is not a viable option, allow for the recommendation of fertilitysparing mechanisms of therapy.

\section{METHODS}

A prospective pilot study of adult patients with confirmed diagnosis of complex hyperplasia with atypia or EEC during August 2017 through July 2018 was conducted. Further inclusion criteria required that patients must be English-speaking, undergoing hysterectomy with 1 of 2 gynecologic oncologists, eligible for contrast-enhanced 3-tesla MRI scan (eg, no pacemaker or defibrillator), comfortable undergoing MRI scan with/without oral sedation, have less than severely compromised kidney function (ie, glomerular filtration rate of $\geq 30 \mathrm{~mL} / \mathrm{min} /$ $1.73 \mathrm{~m}^{2}$ ), and able to undergo contrast-enhanced MRI scan at a single medical center 7 days prior to scheduled hysterectomy. Patients who presented for their MRI scan prior to their scheduled hysterectomy were provided with a $\$ 50$ gift card. The study was approved by the local institutional review board.

Following MRI scan, a radiologist checked image quality to determine potential need for a rescan. Images were then reviewed by 3 separate radiologists (with 2-10 years of experience depending on time of enrollment) to determine TD (recorded in anteriorposterior, medial-lateral, and craniocaudal planes), depth of invasion (disruption of the junctional zone), and myometrial thickness (recorded thickness at an adjacent noninvolved segment). All TD and MI measurements were recorded in millimeters. Image quality as well as TD and MI image confidence were recorded on a scale from 1 to 3 (poor, moderate, excellent) by all 3 radiologists. Data on time of image acquisition relative to contrast injection were not collected. Following hysterectomy, chart review was performed to determine TD and MI as recorded by pathology.

Basic descriptive statistics including counts, percentages, and means were used to summarize patient characteristics and TD and MI measurements. MI was calculated based on depth of invasion/myometrial thickness $\times 100 \%$. The largest TD and MI reported by pathology for each patient was used as the gold standard for comparison to determine if radiologic measurements were within $5 \mathrm{~mm}$ for largest TD (regardless of plane in which measurement was taken) and within 5\% for MI. Additionally, the radiologist's ability to predict superficial $(<50 \%)$ versus deep ( $\geq 50 \%$ ) MI was examined. Sensitivity, specificity, and accuracy were calculated per standard formulas for MI. Given the size of our study population, no P-values were calculated. All analyses were conducted using SAS 9.4 (SAS Institute Inc., Cary, NC).

\section{RESULTS}

A total of 12 patients were enrolled and underwent contrast-enhanced preoperative MRI. Patients were predominately white $(91.7 \%)$, had mean age of 58.8 years (range: $37.3-70.8$ years; only 1 patient was under 40 years old), and had mean body mass index of 35.6 
$\mathrm{kg} / \mathrm{m}^{2}$. Those enrolled had similar histologic grade and stage, with $91.7 \%$ of patients presenting with International Federation of Obstetrics and Gynecology (FIGO) grade 1 tumors and $75.0 \%$ of patients presenting with stage 1a uterine cancer, respectively. On average, patients received their MRI scan and surgery within 33.3 days and 38.7 days of diagnosis, respectively.

In comparison to the largest pathology-reported $\mathrm{TD}$, at least 1 radiologist was able to accurately predict largest TD within $5 \mathrm{~mm}$ for $41.7 \%$ of patients. While 2 radiologists were able to predict largest TD for 3 patients, all radiologists were not able to predict largest TD for any 1 patient. One radiologist was able to predict largest TD within $5 \mathrm{~mm}$ for 5 patients. Ultimately, large variability in measurements existed among radiologists (Figure 1).

Similarly, in comparison to the pathology reported MI, at least 1 radiologist was able to predict no MI or MI within $5 \%$ for $50 \%$ of patients. All radiologists were able to predict no MI for 1 patient, with 2 radiologists able to predict MI for 1 patient (Figure 2). All radiologists were not able to predict MI for any 1 patient. Even so, all radiologists were able to predict superficial MI or deep MI for $75 \%$ of patients, with a kappa statistic of 0.65 indicating high interrater agreement (Figure 3). Use of MRI had greater sensitivity, specificity, and accuracy when predicting deep MI (Table 1).

Radiologists identified a mean MRI image quality of 2.3 (on 1-3 scale). Overall, radiologists were relatively confident in TD and MI measurements, expressing a 2.6 and 2.3 level of confidence, respectively.

\section{DISCUSSION}

This pilot study aimed to determine if MRI could precisely measure TD and MI among patients with EEC. Unfortunately, we identified that all radiologists were not able to predict largest TD within $5 \mathrm{~mm}$ of pathologyreported measurements for any single patient. To our knowledge, limited studies have evaluated the ability to precisely measure TD through MRI. A previous study by Bourgioti et al used preoperative TD measured through MRI to predict MI, suggesting that larger TD is associated with greater odds of deep MI that may potentially require surgical management. ${ }^{19}$ Ytre-Hauge and colleagues also correlated greater anteroposterior TD with deep MI, finding low interobserver variability $(0.78-0.85)$ for all TD measurements. ${ }^{17}$ However, we observed large variability among our radiologists, as is seen in Figure 1.

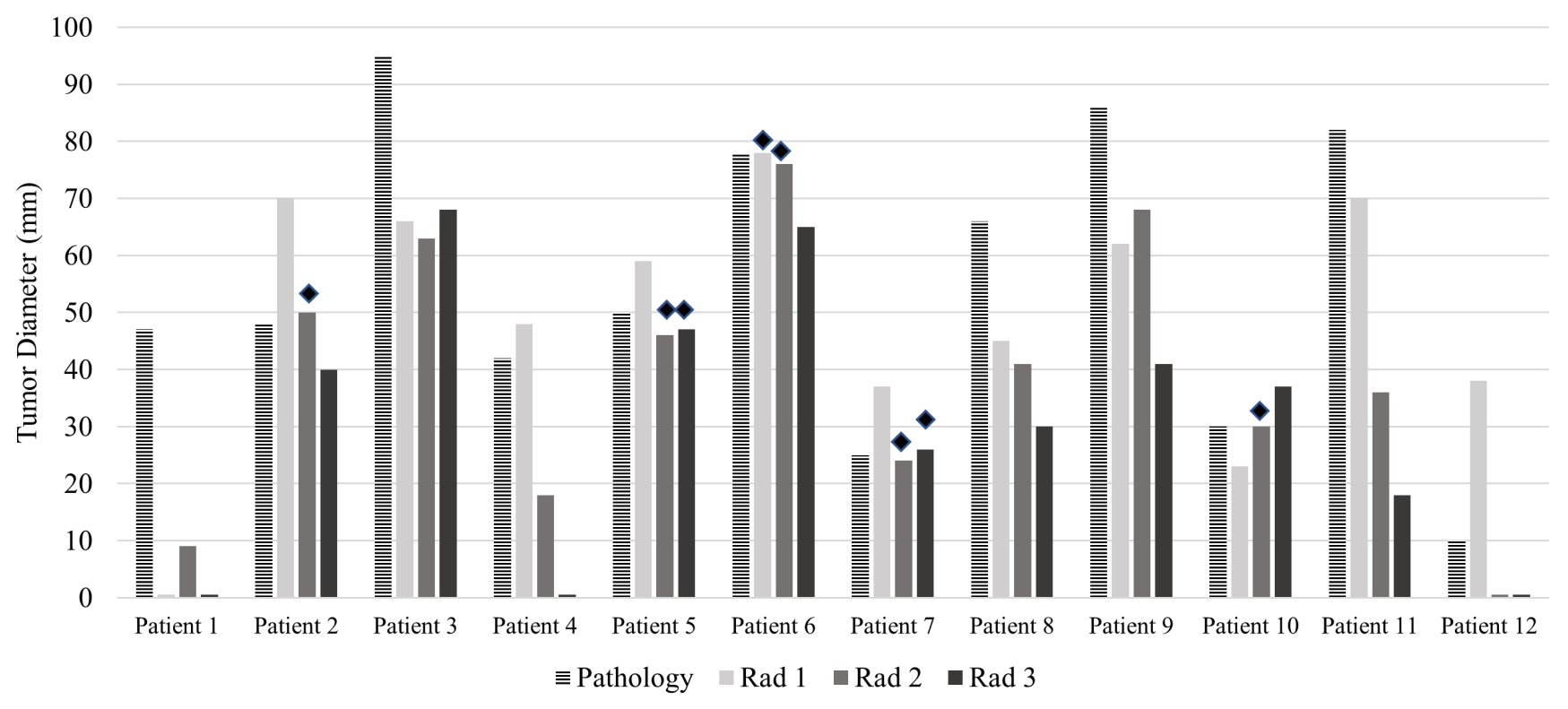

Figure 1. Prediction of largest tumor diameter within $5 \mathrm{~mm}$. Largest tumor diameter as determined by pathology is considered the gold standard (horizontal stripped bar) for each patient. If the radiologist's largest tumor diameter measurement was within $5 \mathrm{~mm}$ of pathology, their measurement is reported with a diamond above the bar. Note: Measurements of zero for tumor diameter are recorded as $0.5 \mathrm{~mm}$ for graphing purposes. 


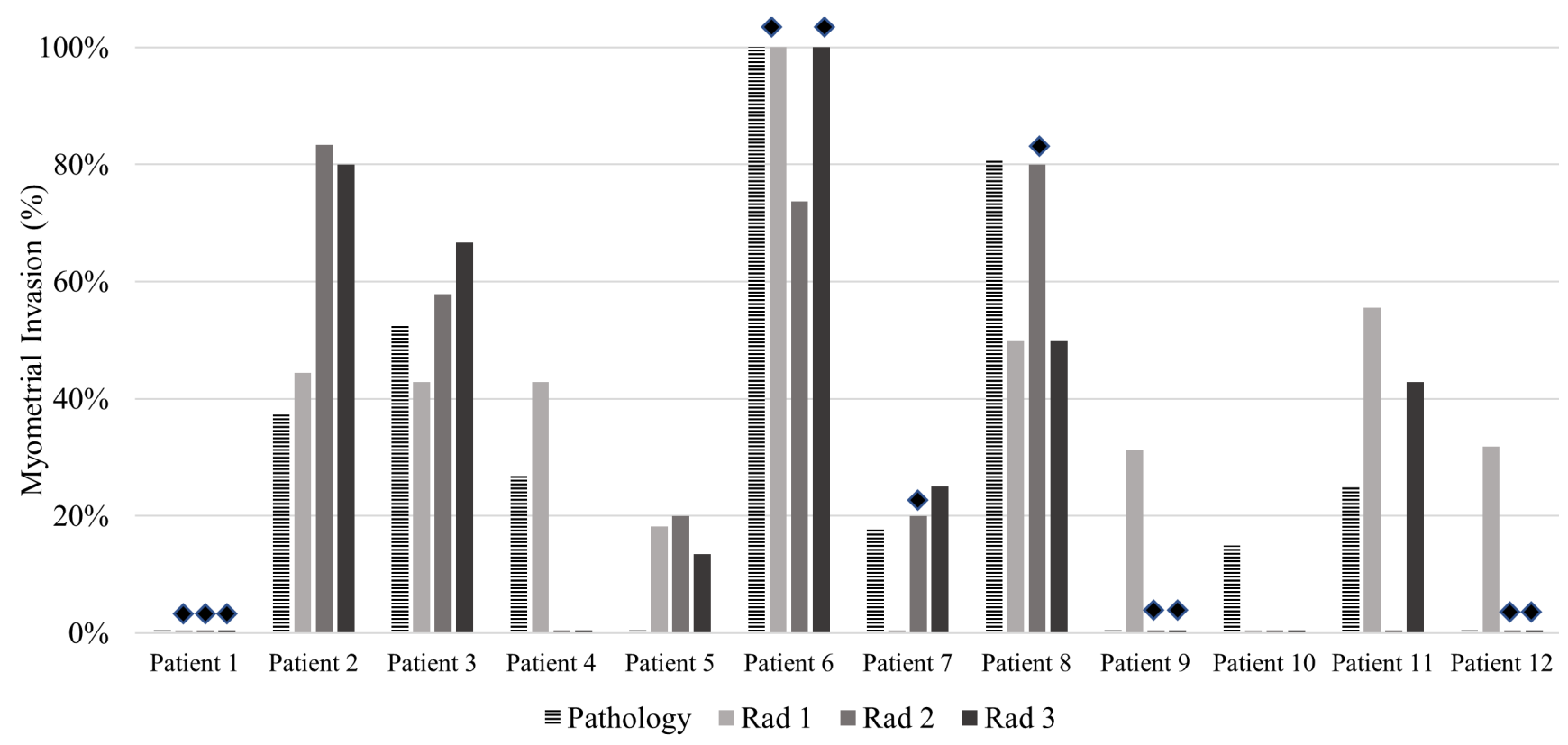

Figure 2. Prediction of myometrial invasion within 5\%. Percentage of myometrial invasion as determined by pathology is considered the gold standard (horizontal stripped bar) for each patient. If the radiologist's myometrial invasion measurement was within 5\% of pathology, their measurement is reported with a diamond above the bar. Note: Measurements for no myometrial invasion are recorded as $0.5 \%$ for graphing purposes.

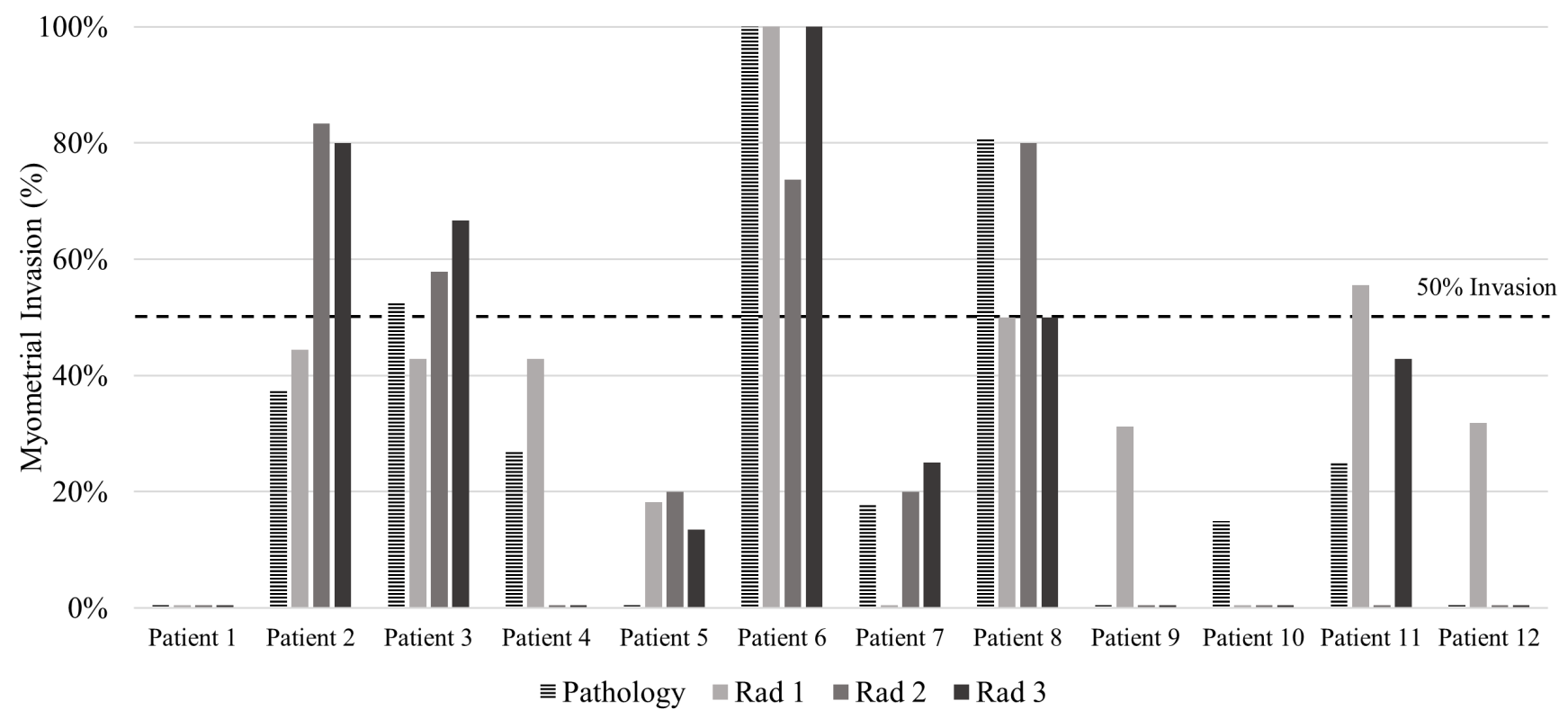

Figure 3. Prediction of superficial versus deep myometrial invasion. The dashed target line is at $50 \%$ invasion. Again, pathology-measured myometrial invasion is considered the gold standard (horizontal stripped bar) for each patient. Kappa statistic for interrater agreement was 0.65. 
Table 1. Sensitivity, Specificity, and Accuracy Among Radiologists in Predicting Myometrial Invasion

\begin{tabular}{|c|c|c|c|c|}
\hline Noninvasive & Radiologist \#1 & Radiologist \#2 & Radiologist \#3 & Average $^{a}$ \\
\hline Sensitivity & $75 \%$ & $62.5 \%$ & $75 \%$ & $70.8 \%$ \\
\hline Specificity & $25 \%$ & $75 \%$ & $75 \%$ & $58.3 \%$ \\
\hline Accuracy & $58.3 \%$ & $66.7 \%$ & $75 \%$ & $66.7 \%$ \\
\hline$<50 \%$ & Radiologist \#1 & Radiologist \#2 & Radiologist \#3 & Average $^{a}$ \\
\hline Sensitivity & $40 \%$ & $20 \%$ & $40 \%$ & $33.3 \%$ \\
\hline Specificity & $42.8 \%$ & $85.7 \%$ & $85.7 \%$ & $71.4 \%$ \\
\hline Accuracy & $41.7 \%$ & $58.3 \%$ & $66.7 \%$ & $55.6 \%$ \\
\hline$\geq 50 \%$ & Radiologist \#1 & Radiologist \#2 & Radiologist \#3 & Average $^{a}$ \\
\hline Sensitivity & $66.7 \%$ & $100 \%$ & $100 \%$ & $88.9 \%$ \\
\hline Specificity & $88.9 \%$ & $88.9 \%$ & $88.9 \%$ & $88.9 \%$ \\
\hline Accuracy & $83.3 \%$ & $91.7 \%$ & $91.7 \%$ & $88.9 \%$ \\
\hline
\end{tabular}

${ }^{a}$ Represents the average of all three radiologists for sensitivity, specificity, and accuracy.

Our study identified that all radiologists were not able to predict MI within 5\% of pathology-reported measurements for any single patient. Even so, all were able to predict superficial versus deep MI as identified by pathology in $75 \%$ of patients, and all showed greater sensitivity, specificity, and accuracy when predicting deep MI. In looking for deep MI with MRI, Husby et al reported only fair interrater agreement $(\kappa: 0.32),{ }^{20}$ whereas our interrater agreement for deep MI was higher ( $\kappa$ : 0.65). Previous studies also have explored the ability to measure superficial versus deep MI through MRI. ${ }^{13-16}$ A study by Ryoo et al found that $25.8 \%$ of patients had incorrect MI prediction by MRI, with deep MI patients of increased age and with increased number of previous deliveries being more likely to have an incorrect prediction. ${ }^{13}$ The authors concluded that precise or accurate assessment of MI through MRI is difficult to predict and should be cautiously interpreted and that tumor and patient characteristics may further impact ability to predict MI. ${ }^{13}$ Potentially, such factors also may impact ability to predict accurate TD. Antonsen et al suggested MRI should not replace surgical staging but could potentially be used to rule out deep MI for those who can't or don't want to undergo surgical staging. ${ }^{15}$

Endometrial cancer is one of the few cancers with increasing incidence ${ }^{3}$ and is associated with increased surgical morbidity and all-cause mortality, especially among those who are morbidly obese. ${ }^{21,22}$ As previously identified, obesity is increasing among younger women, ${ }^{5}$ with adipose tissue creating a hyperestrogen state, thereby putting these women at increased risk of developing endometrial cancer earlier in life. ${ }^{21}$ Thus, more fertility-sparing and nonsurgical options for endometrial hyperplasia and endometrial cancer are needed for patients. Ultimately, while our findings demonstrated that the ability to precisely measure MI and TD through MRI was limited, the ability to measure superficial versus deep MI was evident. Potentially, use of MRI may guide clinicians on care options among younger women seeking fertility-sparing or nonsurgical treatments. Additionally, it may help to guide care for women who wish to avoid lymphadenectomy. Further study is warranted.

This pilot had several limitations; one limitation in and of itself was that the scarcity of prior evidence limited our ability to solidly recommend MRI for preoperative risk assessment. While we aimed to enroll 3 times as many patients, we had difficulties enrolling (patients didn't want to undergo additional test, drive to testing site, etc) and scheduling (eg, MRI machine was unavailable prior to surgery) patients. Even so, among the study's 12 patients, we saw a large degree of variation among radiologic measurements that would most likely have existed regardless of an increased sample size. The variability in measurements could be due to the experience level of the participating radiologists (ie, 2-10 years) and how frequently they read pelvic MRIs in their daily practice (per our radiologists, 
pelvic MRIs are relatively uncommon within our health system and tend to be read by those with greater experience).

Previous studies have shown an association with apparent diffusion coefficient values and endometrial carcinomas. However, we did not collect these values. $^{20}$ Additionally, the majority of patients within our study were stage 1a, and it is unknown if radiologic measurement variability would remain in higher-stage patients. However, by having a similar patient population, variability among radiologist measurements should have been minimal. Lastly, we didn't determine whether radiologists were able to predict smaller TD dimensions, as largest TD or primary TD was used for identifying risk in previous retrospective studies. ${ }^{2,10,11}$

Given the variation between radiologic assessments, it is difficult to recommend preoperative MRI as a basis for precisely measuring tumor diameter and myometrial invasion. However, while huge variation existed in predicting MI within 5\%, radiologists may be able to predict superficial versus deep MI. Predicting superficial versus deep MI preoperatively through contrast-enhanced MRI might help providers better manage endometrioid endometrial cancer in patients who are not eligible for surgery or are seeking fertility-sparing treatments.

\section{Patient-Friendly Recap}

- Patients with endometrioid endometrial cancer, a type of uterine cancer, may be candidates for noninvasive treatment that preserves future ability to bear children.

- The authors tested whether radiologists were able to accurately measure tumor size and gauge patient risk using MRI.

- Given the variation found among radiologic measurements, it is difficult to recommend preoperative MRI as a tool for measuring endometrioid endometrial cancer status.

- Radiologists were better at broadly distinguishing mild from deep cancerous invasion, indicating this tool may be useful in patients opposed to surgical therapy.

\section{Acknowledgments}

We would like to thank Danielle M. Greer, PhD, and Salik Nazeer, MD, both of Aurora Health Care at time of study, for substantial project design and conception and Pamela Aoys and Lisa Robinson, both of Aurora Health Care, for their contributions throughout the project. We also thank all those who supported this study as part of their routine work and, specifically, Kellie Rozumalski of Aurora Health Care for ensuring MRIs were appropriately scheduled.

\section{Author Contributions}

Study design: all authors. Data acquisition or analysis: all authors. Manuscript drafting: Dickson Michelson, Kram, Heslin. Critical revision: Baugh, Bamra, Hu, Shukla, Kamelle.

\section{Conflicts of Interest}

None.

\section{Funding Sources}

This research was supported by an Aurora Research Institute Oncology Research Award, funded by the Vince Lombardi Cancer Foundation (Elm Grove, WI) and other generous donors.

\section{References}

1. American Cancer Society. Key statistics for endometrial cancer. Last reviewed 2020 Jan 8. https://www.cancer.org/ cancer/endometrial-cancer/about/key-statistics.html. Accessed April 9, 2020.

2. Mariani A, Dowdy SC, Cliby WA, et al. Prospective assessment of lymphatic dissemination in endometrial cancer: a paradigm shift in surgical staging. Gynecol Oncol. 2008;109:11-8. CrossRef

3. American Cancer Society Cancer Statistics Center. Uterine corpus. https://cancerstatisticscenter.cancer.org/\#!/cancer-site/ Uterine\%20corpus. Accessed July 1, 2019.

4. Van Arsdale A, Miller DT, Kuo DY, Isani S, Sanchez L, Nevadunsky NS. Association of obesity with survival in patients with endometrial cancer. Gynecol Oncol. 2019;154:156-62. CrossRef

5. Gonthier C, Walker F, Luton D, Yazbeck C, Madelenat P, Koskas M. Impact of obesity on the results of fertility-sparing management for atypical hyperplasia and grade 1 endometrial cancer. Gynecol Oncol. 2014;133:33-7. CrossRef

6. Navarria I, Usel M, Rapiti E, et al. Young patients with endometrial cancer: How many could be eligible for fertilitysparing treatment? Gynecol Oncol. 2009;114:448-51. CrossRef

7. Society of Gynecologic Oncology. SGO Clinical Practice Statement: the role of sentinel lymph node mapping in endometrial cancer. Issued 2015 Nov. https:/www.sgo.org/ clinical-practice/guidelines/the-role-of-sentinel-lymph-nodemapping-in-endometrial-cancer/. Accessed June 28, 2019.

8. Rossi EC, Kowalski LD, Scalici J, et al. A comparison of sentinel lymph node biopsy to lymphadenectomy for endometrial cancer staging (FIRES trial): a multicentre, prospective, cohort study. Lancet Oncol. 2017;18:384-92. CrossRef

9. Bodurtha Smith AJ, Fader AN, Tanner EJ. Sentinel lymph node assessment in endometrial cancer: a systematic review and metaanalysis. Am J Obstet Gynecol. 2017;216:459-76.e10. CrossRef

10. Cox Bauer CM, Greer DM, Kram JJF, Kamelle S. Tumor diameter as a predictor of lymphatic dissemination in endometrioid endometrial cancer. Gynecol Oncol. 2016;141:199-205. $\underline{\text { CrossRef }}$ 
11. Greer DM, Kram JJ, Cox Bauer CM, Kamelle SA. Robustness of a newly proposed risk schema for lymphatic dissemination in endometrioid endometrial cancer. (abstr.) $J$ Patient Cent Res Rev. 2017;4:262. CrossRef

12. Tuomi T, Pasanen A, Leminen A, Bützow R, Loukovaara M. Prediction of lymphatic dissemination in endometrioid endometrial cancer: comparison of three risk-stratification models in a single-institution cohort. Gynecol Oncol. 2017;144:510-4. CrossRef

13. Ryoo UN, Choi CH, Yoon JY, et al. MR imaging in endometrial carcinoma as a diagnostic tool for the prediction of myometrial invasion and lymph node metastasis. Cancer Res Treat. 2007;39:165-70. CrossRef

14. Torricelli P, Ferraresi S, Fiocchi F, et al. 3-T MRI in the preoperative evaluation of depth of myometrial infiltration in endometrial cancer. AJR Am J Roentgenol. 2008;190:489-95. CrossRef

15. Antonsen SL, Jensen LN, Loft A, et al. MRI, PET/CT and ultrasound in the preoperative staging of endometrial cancer - a multicenter prospective comparative study. Gynecol Oncol. 2013;128:300-8. CrossRef

16. Rodriguez-Trujillo A, Martínez-Serrano MJ, Martínez-Román $\mathrm{S}$, et al. Preoperative assessment of myometrial invasion in endometrial cancer by 3D ultrasound and diffusion-weighted magnetic resonance imaging: a comparative study. Int $J$ Gynecol Cancer. 2016;26:1105-10. CrossRef
17. Ytre-Hauge S, Husby JA, Magnussen IJ, et al. Preoperative tumor size at MRI predicts deep myometrial invasion, lymph node metastases, and patient outcome in endometrial carcinomas. Int J Gynecol Cancer. 2015;25:459-66. CrossRef

18. Haldorsen IS, Salvesen HB. What is the best preoperative imaging for endometrial cancer? Curr Oncol Rep. 2016;18(4):25. CrossRef

19. Bourgioti C, Chatoupis K, Tzavara C, Antoniou A, Rodolakis A, Moulopoulos LA. Predictive ability of maximal tumor diameter on MRI for high-risk endometrial cancer. Abdom Radiol (NY). 2016;41:2484-95. CrossRef

20. Husby JA, Salvesen ØO, Magnussen IJ, et al. Tumour apparent diffusion coefficient is associated with depth of myometrial invasion and is negatively correlated to tumour volume in endometrial carcinomas. Clin Radiol. 2015;70:487-94. CrossRef

21. Bouwman F, Smits A, Lopes A, et al. The impact of BMI on surgical complications and outcomes in endometrial cancer surgery - an institutional study and systematic review of the literature. Gynecol Oncol. 2015;139:369-76. CrossRef

22. Secord AA, Hasselblad V, Von Gruenigen VE, et al. Body mass index and mortality in endometrial cancer: A systematic review and meta-analysis. Gynecol Oncol. 2016;140(1):184-190. CrossRef

(C) 2020 Aurora Health Care, Inc. 